\title{
Synthesis and Performance Evaluation of a Betaine Surfactant
}

\author{
ZHANG Rongjun ${ }^{1,2,3, a^{*}}$ ZHOU Zhengpeng ${ }^{3, b}$ WEI Xuegang ${ }^{3, c}$ WEI wei ${ }^{4, d}$ WANG Xiaoke ${ }^{5, \mathrm{e}}$ ZHAO Jinlin ${ }^{6, \mathrm{f}}$ \\ ${ }^{1}$ Xi'an Key Laboratory of Tight oil (Shale oil) Development (Xi'an Shiyou University) \\ ${ }^{2}$ Shaanxi Key Laboratory of Carbon Dioxide Sequestration and Enhanced Oil Recovery \\ ${ }^{3}$ College of Petroleum Engineering, Xi'an Shiyou University Xi'an, Shaanxi, China \\ ${ }^{4}$ Oil Production Plant 11 of PetroChina Changqing Oilfiled Company, Qingyang, Gansu, China \\ ${ }^{5}$ Training Center of PetroChina Changqing Oilfield Company Xi'an, Shaanxi, China \\ ${ }^{6}$ Oil Production Plant 2 of PetroChina Changqing Oilfiled Company Qingyang, Gansu, China
}

\begin{abstract}
Using sodium bisulfite and epichlorohydrin as raw materials, the ring-opening reaction is carried out under the action of acatalyst to produce sodium 3-chloro-2-hydroxypropane sulfonate, followed by dodecyl tertiary amine, isopropanol, sodium hydroxide and 3-chloro-2-hydroxypropane sulfonate sodium as raw materials, under certain conditions to synthesize dodecyl hydroxy sulfobetaine surfactant. The surface tension, interfacial tension, salt resistance and emulsification properties of the synthesized surfactants were tested and evaluated. The experimental results show that the surfactant has a low critical micelle concentration and surface tension; the interfacial tension decreases with the increase of surfactant concentration. When the concentration is greater than or equal to $0.3 \%$, the interfacial tension reaches $10^{-2}$ level; the dodecyl hydroxy sulfobetaine surfactant solution shows good salt resistance; when the concentration of dodecyl hydroxy sulfobetaine surfactant solution is $0.5 \%$, the water separation rate in 120 minutes is the lowest and the emulsification ability is the strongest.
\end{abstract}

\section{INTRODUCTION}

The betaine has a wide range of uses in daily chemical production. Betaine-type surfactants are stable to hard water, have excellent foaming power and wetting power, and have good compatibility with other types of surfactants [1]. The dodecyl hydroxy sultanate is a kind of amphoteric surfactant. Its structure has not only anionic groups, but also cationic groups. Therefore, it has more good properties than other surfactants, such as excellent hard water resistance, electrolyte resistance, low toxicity, good compatibility, mild performance, and easy degradation by organisms. The hydroxy sulfobetaine not only contains all the characteristics and advantages of amphoteric surfactants, but also has numerous advantages such as better emulsification, antistatic and dispersibility. It has been widely used in daily chemical industry, oil displacement, fracturing, acidification and other fields [23]. In this paper, the sodium bisulfite and epichlorohydrin were used as raw materials to produce sodium 3-chloro-2hydroxypropane sulfonate[4]. Then, dodecyl tertiary amine, isopropanol, sodium hydroxide and sodium 3chloro-2-hydroxypropane sulfonate were used as raw materials to synthesize dodecyl hydroxy sulfobetaine surfactant, and the properties of the surfactant solution were studied [5].

\section{EXPERIMENTAL PART}

\subsection{Materials}

Sodium bisulfite, analytical grade, Xi'an chemical reagent factory; epichlorohydrin, analytical grade, Chengdu Kelon chemical reagent factory; sodium hydroxide, analytical grade, Tianjin Hedong District Hongyan Reagent Factory; isopropanol, analysis Pure, Chengdu Kelon Chemical Reagent Factory; calcium chloride, Analytical Pure, Tianjin Shengao Chemical Reagent Co., Ltd.; sodium chloride, Analytical Pure, Tianjin Shengao Chemical Reagent Co., Ltd.; magnesium Chloride, Analytical Pure, Tianjin Sheng Austrian Chemical Reagent Co., Ltd.; potassium chloride, analytical pure, Tianjin Shengao Chemical Reagent Co., Ltd.; crude oil, provided by Changqing No. 1 Oil Production Plant.

\subsection{Instrument}

intelligent magnetic stirrer(ZNCL-GS 190*90, Xi'an Yuhui Co., Ltd.); electric heating constant temperature drying oven(GX-130, Shanghai Dianzhouguang Technology Co., Ltd.); precision power-increasing

\footnotetext{
* Corresponding author: ${ }^{a}$ rongjunzhang@xsyu.edu.cn be-mail: 779601154@qq.com ce-mail: 1633044581@qq.com

de-mail: 263324027@qq.comee-mail r: 691834708@qq.com fe-mail: 669212972@qq.com
} 
electric stirrer(JJ-1, Guohua Electric Co., Ltd.); electric constant temperature water bath (DK-97-IIA, Tianjin Taisite Instrument Co., Ltd.); electronic analytical balance (TP-520H, Xiangyi Balance Instrument Equipment Co., Ltd.); surface tension meter(QBZY-1, Shanghai Fangrui Instrument Factory); rotating droplet interfacial tension meter(XZD-5, Beijing Harco Experimental Instrument Factory).

\subsection{Resolve resolution}

The sodium bisulfite and water are put into three bottles, increasing the temperature and stirring it until the temperature rises to $70^{\circ} \mathrm{C}$ to ensure that the sodium bisulfite is completely dissolved. Then prepare to add epichlorohydrin, ensuring that the temperature has always been kept at $70{ }^{\circ} \mathrm{C}$ for 2 hours. After the dropwise addition, the temperature is increased to $85^{\circ} \mathrm{C}$, after $1.5 \sim$ 2 hours of reaction, cooling it will precipitate white crystal. These white crystals were treated by vacuum filtration, placed in a culture dish and dried in a vacuum drying oven for 48 hours to obtain sodium 3-chloro-2hydroxypropanesulfonate [6]. The reaction equation is shown in Fig. 1.

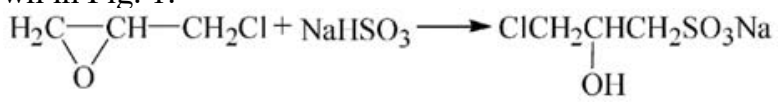

Figure 1. Synthesis of the Sodium 3-chloro-2-hydroxypropane sulfonate

The volume ratio of water to isopropanol is $1: 3$, and then sodium hydroxide and sodium 3-chloro-2hydroxypropane sulfonate are added. The temperature is raised to $90{ }^{\circ} \mathrm{C}$ and the sodium 3-chloro-2hydroxypropanesulfonate is completely dissolved. At this time, the dodecyl tertiary amine is added, reaction time is 4 hours, and then vacuum distillation and extraction are carried out. The reaction equation is shown in Fig. 2<smiles>CCC[N+](C)(CC)CC(O)C[Se+]#[Os]</smiles>

Figure 2. Synthesis of the Sodium 3-chloro-2-hydroxypropane sulfonate

\section{PERFORMANCE EVALUATION}

\subsection{Surface tension evaluation}

$0.001 \%, 0.005 \%, 0.01 \%, 0.05 \%, 0.1 \%, 0.2 \%, 0.3 \%, 0.4 \%$ and $0.5 \%$ dodecyl hydroxy sulfobetaine surfactant solution were prepared with distilled water at room temperature, and then the surface tension was measured by platinum plate method. In order to reduce the error, three experiments were carried out for each concentration sample, and the mode number of the three experimental results was taken as the final experimental data.

\subsection{Interfacial tension evaluation}

$0.05 \%, 0.1 \%, 0.2 \%, 0.3 \%, 0.4 \%$ and $0.5 \%$ dodecyl hydroxy sulfobetaine surfactant solutions were prepared with distilled water, and then the surface tension of kerosene was measured with XZD-5 rotating droplet interfacial tension meter at a fixed temperature of $30{ }^{\circ} \mathrm{C}$.

\subsection{Salt resistance evaluation}

$0.1 \%$ dodecyl hydroxy sulfobetaine surfactant solution was prepared with distilled water, and then potassium chloride, sodium chloride, anhydrous calcium chloride and magnesium chloride with different mineralization degrees were added respectively. The surface tension was measured by a platinum plate method, and the turbidity degree of the solution was observed.

\subsection{Emulsification ability evaluation}

$0.1 \%, 0.2 \%, 0.3 \%, 0.4 \%$ and $0.5 \%$ dodecyl hydroxy sulfobetaine surfactant solutions were prepared with distilled water and mixed with crude oil at a volume ratio of $1: 1$. The water evolution rate was measured with a stopwatch at $40{ }^{\circ} \mathrm{C}$ and $50{ }^{\circ} \mathrm{C}$ for 2 hours.

\section{RESULTS \& DISCUSSION}

\subsection{Surface tension}

It can be concluded from Table I that the surface tension of dodecyl hydroxy sulfobetaine surfactant solution decreases rapidly as the concentration increases, and then decreases slowly, and tends to be stable when it drops to a certain value. It can be concluded from the table that the critical micelle concentration of dodecyl hydroxy sulfobetaine surfactant is about $0.1 \%$, and the surface tension corresponding to critical micelle concentration point is $29.8 \mathrm{mN} / \mathrm{m}$. The results showed that the critical micelle concentration and surface tension of the surfactant were low.

TABLE I. SURFACE TENSION OF DODECYL HYDROXY SULFOBETAINE SURFACTANT SOLUTIONS WITH DIFFERENT CONCENTRATIONS

\begin{tabular}{|c|c|c|c|c|}
\hline \multirow{2}{*}{$\begin{array}{l}\text { Concentration } \\
(\mathrm{g} / \mathbf{1 0 0 \mathrm { ml } )}\end{array}$} & \multicolumn{4}{|c|}{ Surface tension $(\mathbf{m N} / \mathbf{m})$} \\
\hline & $\begin{array}{c}\text { the first } \\
\text { time }\end{array}$ & $\begin{array}{l}\text { the second } \\
\text { time }\end{array}$ & $\begin{array}{l}\text { the third } \\
\text { time }\end{array}$ & $\begin{array}{c}\text { average } \\
\text { value }\end{array}$ \\
\hline 0.001 & 51.3 & 51.3 & 51.2 & 51.3 \\
\hline 0.005 & 46.4 & 46.5 & 46.4 & 46.4 \\
\hline 0.01 & 40.3 & 40.3 & 40.3 & 40.3 \\
\hline 0.05 & 31.2 & 31.1 & 31.1 & 31.1 \\
\hline 0.1 & 29.8 & 29.9 & 29.8 & 29.8 \\
\hline 0.2 & 29.5 & 29.5 & 29.5 & 29.5 \\
\hline
\end{tabular}




\begin{tabular}{|c|c|c|c|c|}
\hline \multirow{2}{*}{$\begin{array}{c}\text { Concentration } \\
(\mathbf{g} / \mathbf{1 0 0 m l})\end{array}$} & \multicolumn{4}{|c|}{ Surface tension (mN/m) } \\
\cline { 2 - 5 } & $\begin{array}{c}\text { the first } \\
\text { time }\end{array}$ & $\begin{array}{c}\text { the second } \\
\text { time }\end{array}$ & $\begin{array}{c}\text { the third } \\
\text { time }\end{array}$ & $\begin{array}{c}\text { average } \\
\text { value }\end{array}$ \\
\hline 0.3 & 29.5 & 29.5 & 29.5 & 29.5 \\
\hline 0.4 & 30.1 & 30 & 30 & 30 \\
\hline 0.5 & 30 & 30 & 30 & 30 \\
\hline
\end{tabular}

\subsection{Interfacial tension}

At $30{ }^{\circ} \mathrm{C}$, it can be concluded from Table II when the concentration of dodecyl hydroxy sulfobetaine surfactant solution is greater than or equal to $0.1 \%$, the interfacial tension with kerosene is lower than $2 \times 10-1 \mathrm{mN} / \mathrm{m}$. When the concentration of dodecyl hydroxy sulfobetaine surfactant solution is greater than or equal to $0.3 \%$, the interfacial tension with kerosene reaches the order of 10-2 level. With the increase of surfactant concentration, the interfacial tension with kerosene shows a downward trend, and the downward trend becomes stable after the concentration is higher than $0.1 \%$. The change trend of surface tension and interfacial tension of dodecyl hydroxy sulfobetaine surfactant is basically the same.

TABLE II. INTERFACIAL TENSION OF DODECYL HYDROXY

SULFOBETAINE SURFACTANT SOLUTIONS WITH DIFFERENT CONCENTRATIONS

\begin{tabular}{|c|c|c|c|}
\hline $\begin{array}{c}\text { Concentration } \\
(\mathbf{g} / \mathbf{1 0 0 m l})\end{array}$ & $\begin{array}{c}\text { Droplet } \\
\text { diameter } \\
(\mathbf{m m})\end{array}$ & $\begin{array}{c}\text { Rotation } \\
\text { speed }(\boldsymbol{r}) \\
\text { minute })\end{array}$ & $\begin{array}{c}\text { Interfacial } \\
\text { tension } \\
(\mathbf{m N} / \mathbf{m})\end{array}$ \\
\hline 0.05 & 2.5 & 6950 & 0.567 \\
\hline 0.1 & 2.5 & 3950 & 0.183 \\
\hline 0.2 & 2.5 & 3380 & 0.134 \\
\hline 0.3 & 2.3 & 3160 & 0.091 \\
\hline 0.4 & 2.3 & 2810 & 0.072 \\
\hline 0.5 & 2.2 & 2530 & 0.059 \\
\hline
\end{tabular}

\subsection{Salt resistance}

It can be seen from Table III that with the increase of salinity of the four salts, the solution is always transparent, indicating that the solution of dodecyl hydroxy sulfobetaine surfactant has good solubility with the four salts. With the increase of the salinity of the four salts, the surface tension of the four salts showed an upward trend. The rising trend of sodium salt, potassium salt and magnesium salt was first rapid and then slow, while the rising trend of calcium salt was relatively stable. When the salinity of four salts reached $100000 \mathrm{ppm}$, the surface tension change rate was about $10 \%$. Therefore, with the increase of salinity of four salts, the dodecyl hydroxy sulfobetaine surfactant solution showed good salt resistance performance.

TABLE III. SALT RESISTANCE OF SURFACE TENSION OF 0.1\% DODECYL HYDROXY SULFOBETAINE SURFACTANT SOLUTION

\begin{tabular}{|c|c|c|c|c|}
\hline \multirow{2}{*}{$\begin{array}{c}\text { Mineralization } \\
\text { degree } \\
\text { (ppm) }\end{array}$} & \multicolumn{4}{|c|}{ Surface tension (mN/m) } \\
\cline { 2 - 5 } & $\mathbf{N a}^{+}$ & $\boldsymbol{K}^{+}$ & $\mathbf{C a}^{\mathbf{+}}$ & $\boldsymbol{M g}^{\mathbf{2}^{+}}$ \\
\hline 1000 & 29.8 & 29.8 & 29.8 & 29.8 \\
\hline 2000 & 30.2 & 30.2 & 29.9 & 29.9 \\
\hline
\end{tabular}

\begin{tabular}{|c|c|c|c|c|}
\hline \multirow{2}{*}{$\begin{array}{c}\text { Mineralization } \\
\text { degree } \\
\text { (ppm) }\end{array}$} & \multicolumn{4}{|c|}{ Surface tension $(\mathbf{m N} / \mathbf{m})$} \\
\cline { 2 - 5 } & $\mathbf{N a}^{+}$ & $\boldsymbol{K}^{+}$ & $\mathbf{C a}^{2+}$ & $\mathbf{M g}^{2+}$ \\
\hline 5000 & 30.9 & 30.4 & 30.1 & 30.1 \\
\hline 10000 & 31.1 & 30.8 & 30.1 & 30.6 \\
\hline 20000 & 31.4 & 31.2 & 30.3 & 31.2 \\
\hline 50000 & 32.2 & 32.1 & 30.7 & 32.1 \\
\hline 100000 & 33.1 & 33 & 32 & 32.7 \\
\hline
\end{tabular}

\subsection{Emulsification}

At $50{ }^{\circ} \mathrm{C}$, it can be seen from Fig. 3 that with the extension of time, more and more water will be precipitated from different concentrations of dodecyl hydroxy sulfobetaine surfactant solution. With the increase of the concentration, the water separation rate becomes lower and lower, but the water separation rate of different concentrations for 120 minutes is lower than $40 \%$. The water separation rate of $0.1 \%$ dodecyl hydroxy sulfobetaine surfactant solution reached about $37 \%$ in 120 minutes, which was significantly higher than other concentrations, so the emulsifying ability was the worst when the concentration was $0.1 \%$. The concentration of $0.5 \%$ dodecyl hydroxy sulfobetaine surfactant solution has the lowest water evolution rate, and the emulsification effect is the strongest.

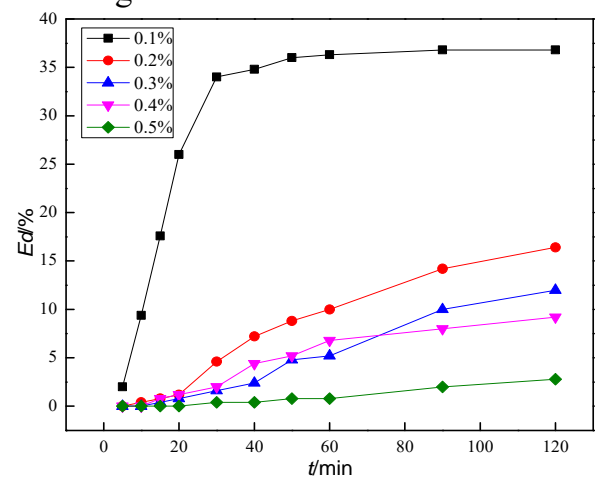

Figure 3. Water splitting rate of dodecyl hydroxy sulfobetaine surfactant solution with $50{ }^{\circ} \mathrm{C}$

At $60{ }^{\circ} \mathrm{C}$, it can be seen from Fig. 4 that with the extension of time, more and more water will be precipitated from different concentrations of dodecyl hydroxy sulfobetaine surfactant solution. With the increase of the concentration, the water precipitation rate becomes lower and lower, but the water precipitation rate of different concentrations for 120 minutes is less than $40 \%$. The water separation rate of $0.1 \%$ dodecyl hydroxy sulfobetaine surfactant solution in 120 minutes was significantly higher than that of other concentrations, so the emulsifying ability was the worst when the concentration was $0.1 \%$. The concentration of $0.5 \%$ dodecyl hydroxy sulfobetaine surfactant is the lowest, and the emulsifying ability is the strongest. 


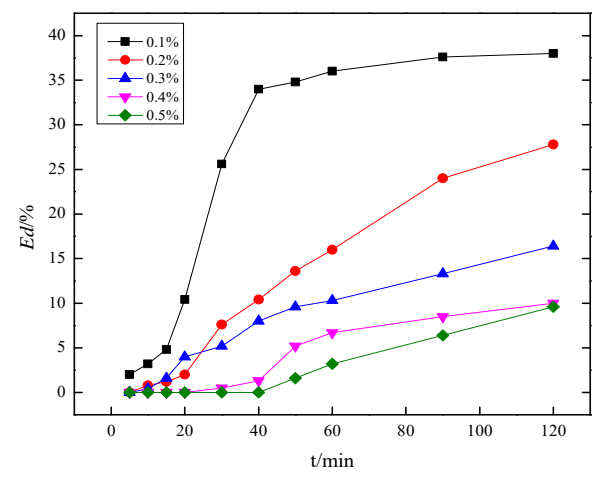

Figure 4. Water splitting rate of dodecyl hydroxy sulfobetaine surfactant solution with $60^{\circ} \mathrm{C}$

Compared with $50{ }^{\circ} \mathrm{C}$, the water separation rate of the same concentration of dodecyl hydroxy sulfobetaine surfactant solution increased at $60{ }^{\circ} \mathrm{C}$, and the increase rate of $0.5 \%$ dodecyl hydroxy sulfobetaine surfactant solution was the largest, which indicated that temperature had great influence on the emulsifying ability of $0.5 \%$ dodecyl hydroxy sulfobetaine surfactant solution.

\section{CONCLUSIONS}

The optimum synthesis conditions of dodecyl hydroxy sulfobetaine surfactant were as follows: the ratio of isopropanol to water was $3: 1$. The reaction temperature was $90{ }^{\circ} \mathrm{C}$, and the reaction time was 4 hours.

At room temperature, the critical micelle concentration of dodecyl hydroxy sulfobetaine surfactant aqueous solution is about $0.1 \%$, and the surface tension corresponding to critical micelle concentration point is $29.8 \mathrm{mN} / \mathrm{m}$. The results showed that the critical micelle concentration and surface tension of the surfactant were low.

When the concentration of dodecyl hydroxy sulfobetaine surfactant solution is more than $0.1 \%$, the interfacial tension with kerosene is less than $2 \times 10^{-1} \mathrm{mN} / \mathrm{m}$. The interfacial tension decreases with the increase of surfactant concentration. When the concentration is greater than or equal to $0.3 \%$, the interfacial tension reaches $10^{-2}$ level.

With the increase of salinity of $\mathrm{Na}^{+}, \mathrm{K}^{+}, \mathrm{Mg}^{2+}$ and $\mathrm{Ca}^{2+}$, the surface tension of dodecyl hydroxy sulfobetaine surfactant solution is always transparent, and the surface tension is increasing. When the salinity of four salts reaches $100000 \mathrm{ppm}$, the surface tension change rate is about $10 \%$, which indicates that the solution of dodecyl hydroxy sulfobetaine surfactant has good solubility with the four salts, and all show good salt resistance.

At the same temperature, with the extension of time, more and more water will be precipitated from different concentrations of dodecyl hydroxy sulfobetaine surfactant solution. With the increase of concentration, the water evolution rate becomes lower and lower, but the water evolution rate is less than $40 \%$ in 120 minutes. At different temperatures, the water evolution rate of the same concentration of dodecyl hydroxy sulfobetaine surfactant solution increases with the increase of temperature. When the concentration of dodecyl hydroxy sulfobetaine surfactant solution is $0.1 \%$, the water separation rate is significantly higher than other concentrations, so the emulsifying ability is the worst when the concentration is $0.1 \%$. The $0.5 \%$ dodecyl hydroxy sulfobetaine surfactant solution has the lowest water separation rate for 120 minutes and the strongest emulsification ability.

\section{ACKNOWLEDGMENT}

This work was financially supported by the grants from Open Fund of Shaanxi Key Laboratory of Carbon Dioxide Storage and Enhanced Oil Recovery(YJSYZX20SKF0008).

\section{REFERENCES}

1. Wang, L., Shi, L. L., Lai, X. J., Liu, P., Wang, J. (2018) Preparation of a betaine type gemini surfactant and its performance evaluation as the main agent of foaming agent. Applied Chemical Engineering, 47: 2169-2173.

2. Zhang, R. M., Liu, A. Q., Shan, H. M., Li, R. (2009) Synthesis and performance evaluation of Tetradecyl hydroxysulfobetaine. Chemistry and Bioengineering, 26: 73-76.

3. Li, K., Li, Z. H., Liu, X. L., Chen, L. Y., Wan, Q. S., $\mathrm{Xu}$, R. J. (2018) Performance evaluation of surfactant in B21 well block of Karamay Oilfield. Xinjiang Petroleum Geology, 39: 220-223 + 243.

4. Shi, Y. Y. (2014) Synthesis of sodium 3-chloro-2hydroxypropane sulfonate. Shandong Chemical Engineering, 43: 20-21.

5. Bai, S. B., Wang, Y. W. (2011) Synthesis of betaine surfactant and its application in tertiary oil recovery. Science and technology innovation guide, 19: 49-50.

6. Chen, Y. Y., Ding, W. (2015) C_ Synthesis and interfacial properties of (20-24) alkyl xylene sulfonates. Chemical engineer, 29: 64-68. 